\title{
Dark Matter. Search for a particle?
}

\author{
Zherikhina L.N. ${ }^{1}$, Izmaïlov G.N. ${ }^{2}$, Tskhovrebov A.M. ${ }^{1}$ \\ ${ }^{1}$ P.N. Lebedev Physical Institute of the RAS, Moscow, Russia; \\ ${ }^{2}$ Moscow Aviation Institute (National Research University) Moscow, Russia; \\ E-mail: Izmailov <gizmailov@mail.ru>;
}

In the report we present the contemporary status of Dark Matter particle search in theoretical and experimental aspects. We analyze some direct methods of registration for non-contact interaction between DM particles and a working medium, and propose the scheme of SQUID - paramagnetic absorber as a new detector.

Keywords: Dark Matter particle, non-contact interaction, SQUID.

DOI: $10.18698 / 2309-7604-2015-1-566-576$

In 1933 the Swiss astronomer F. Zwicky published results of observations of the velocity dispersion for eight galaxies in the Coma Cluster [1]. He claimed the discrepancies between observed data and predicted by Newton's gravity theory velocities pointed to an existence of hidden matter, did not registered by optical instruments. To date, evidences of the existence of hidden matter or now called Dark Matter (DM) have become so many that doubts in its obvious are dispersed. Experimental validations include

- observations of the orbital velocities of stars in galaxies;

- distortions of the sky view, caused by DM;

- the shift of hydrogen emission lines of a matter located in intergalactic space;

- kinematic data of stars and gas streams, indicating a massive and dark halos within galaxies;

- contemporary astrophysics and high-energy physics appreciates the data on X-ray emission of galaxies and galaxy clusters. The data obtained from satellites PAMELA, ROSAT, XMM-Newton, Chandra.

Due to non-stop refining estimates the amount of DM in 5-6 times more than baryonic matter, which represents normal outward things for us. Seeing that the Universe's expansion is accelerating at the greatest distances, we have to adopt also "Dark Energy", which acts to drive the expansion, in opposition to gravity. Taking a mass-energy fork as a base, Dark Energy contributes in sum about 14 times greater than the visual matter. (The observable Universe contains $68.3 \%$ Dark Energy, 26.8\% share Dark Matter and only 4.9\% is visible ordinary (baryonic) matter) [https://ru.wikipedia.org/]. 
What is a source of DM? At first sight it is necessary to choose a particle as a DM constituent. So the most adopted is a model of particles, so due to their energy we have ColdDM, WarmDM, HotDM. Features of this model are

- the large number of candidates $(\sim 50)$

- a variety of mechanisms of interaction

- the possibility of mutual transformation of particles DM

- a variety of interaction parameters

But as pranksters are joking: DM particles are not exist in SM family and we cannot see them through a telescope.

Hypothetically a DM particle interacts with a baryonic particle through gravitation or weak nuclear force and can undergo head-on collisions (fig.1). Specific search of particles is carried out in multiplicity of laboratories around the world, using direct and indirect methods of registration (CRESST, DAMA, EDELWEISS, CDMS-II, CoGeNT, KIMS and ANAIS, DEAP, DarkSide, WARP, PICASSO, TPC, DRIFT, SIMPLE). These studies include a considerable number of space experiments (see above), experiments placed in Antarctica (IceCube).

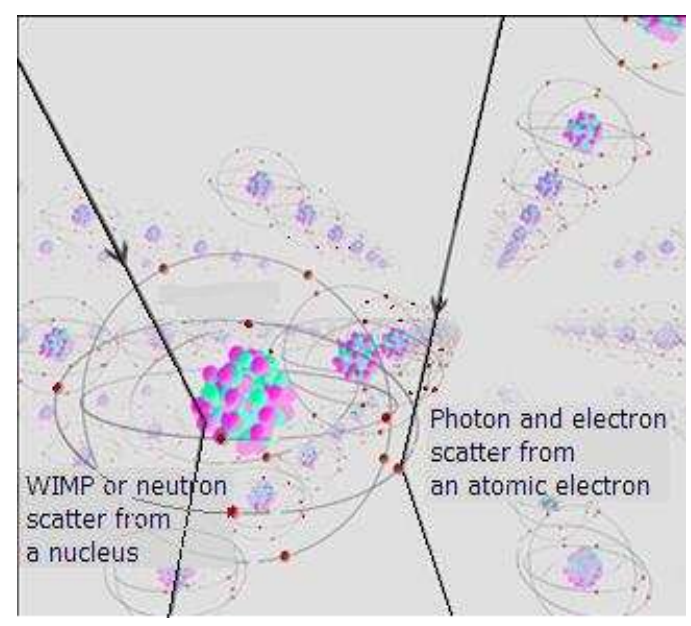

Fig. 1. Scheme of interaction between WIMP and the nucleus of a detector medium. The alternative collision between photon or an electron and the atomic electron is shown. Scatterings of a neutron, an electron and a photon are a noise upon detecting.

According to important cosmological measurements [2-5], we have found the following results:

- $\quad$ only the $1 \%$ of DM is of baryonic kind (black holes, neutron stars, big planets); 
- $\quad$ almost the $30 \%$ is represented by Hot Dark Matter (HDM) [6], made up by relativistic particles, with mass smaller than $30 \mathrm{eV}$, so they cannot clump: the candidate HDM particles are the cosmological neutrinos, created by the Big Bang;

- the remaining $69 \%$ is represented by the so called Cold Dark Matter (CDM) [7], which is made up by non-relativistic particles, with masses comprised between $\mathrm{GeV}$ and $\mathrm{TeV}$.

Due to a large mass and consequently slow velocity their interaction energy takes over kinetic energy and they clump together forming Large Scale Structure, and can be revealed by gravitational effects. But in virtue of the minuscule cross section a CDM particle doesn't interact with a baryonic particle and can't annihilate with its antiparticle. At least a dozen candidates were proposed to represent such particles. The most accredited candidate is the WIMPs (Weakly Interacting Massive Particle), but there are also some other candidates which are very exotic [8]. For their larger abundance, and its non-negligible gravitational effects, the CDM as the part of DM is more analyzed in the modern theoretical and experimental physical research, and we consider just CDM in our paper.

Table 1. The estimates for such particle as WIMP are summarized.

\begin{tabular}{|lc|l|}
\hline Mass & MW $_{W}$ & $10-5000 \mathrm{GeV} / \mathrm{c}^{2}(\sim 10-5000 \mathrm{mP})$ \\
\hline Velocity & $\mathrm{VW}_{W}$ & $\mathbf{1 0}^{5}-\mathbf{1 0}^{6} \mathrm{~m} / \mathrm{sec}$ \\
\hline Density & $\rho_{W}$ & $\mathbf{0 , 3}\left(\mathrm{GeV} / \mathrm{c}^{2}\right) / \mathrm{cm}^{3}$ \\
\hline $\begin{array}{l}\text { Cross-section of } \\
\text { interaction }\end{array}$ & $\sigma_{W}$ & \\
\hline Flux & $\Phi_{W}$ & $\sim 5 * 10^{-10} \mathrm{pbarn}\left(\sim \mathbf{1 0}^{-44} \mathrm{~cm}^{2}\right)$ \\
\hline Electric/Color charge & $1 /\left(\mathrm{cm}^{2} \mathrm{sec}\right)$ \\
\hline
\end{tabular}

The two kinds of particles which best fit these parameters are:

The Heavy Neutralinos, described in the Theory of Supersymmetry (SUSY), which postulates the existence of superpartners of ordinary particles, i.e. new particles whose spins differ by $1 / 2$.

The Lightest Kaluza-Klein Particles (LKP), described in the String Theory, which postulates the existence of extra spatial and temporal dimensions in Universe, inside whom these 
Kaluza-Klein particles (KK) exist as massive excited states, and the lightest of them is the appealing candidate for DM, with mass values between 400-1200 GeV.

Other candidates - neutrinos and axions (with masses $<10 \mathrm{eV} / \mathrm{c}^{2}$ ) are SIMP (Slim Interaction Massless Particles) particles. As they are nearly massless, they move very fast. Some new properties of DM particle are considered in nowadays.

CERN, with its LHC is also included in the search for the mysterious particles (for example, hooperon - neutralino(?) with mass $\sim 20-100 \mathrm{GeV} / \mathrm{c}^{2}$ ) do not belong to the family of the Standard Model particles. Theoreticians guess $\gamma$ - radiation coming from the Galactic Center is caused by interactions between such particles. It is expected that the CERN's CMS detector will register the neutralino, which will be born during the series of clashes in the modernized LHC.

Many conferences, including PIRT, pay attention to discussions of the results obtained in the already established and running installations, the theoretical justifications of methods of research or strategies to explore different observation. Plus a lot of opinions expressed in papers in Nature, Science, Physical Revue, The Astrophysical Journal, ArXiv, etc.

But any DM particle has not revealed yet. Thus, there is a situation in which an object is detected, but its composition is not determinate.

To continue the search we can use another models of DM and DM particles interaction.

$\mathrm{DM}$ is considered from the standpoint of the MOdified Gravitational theory (MOG) and MOdified Newtonian Dynamics (MOND).

It is believed that DM is a quantum defect of the Universe.

It is guessed that DM is a mass generated by extra dimensions.

It is interesting to note that from another astrophysical observations and considerations DM constitutes threads and filaments spreading on dozens of light years. The collision of galaxies, indicating a weak interaction between DM itself, observations and modeling of filament structures substantiate the hydrodynamic approach [11-15].

The overview of results obtained in different experimental setups, points to a significant difference between the spin-dependent SD and the spin-independent SI cross sections of interactions so that $\sigma_{S D} / \sigma_{S I} \sim 10^{8}$ for simple working substances (fig.2). 

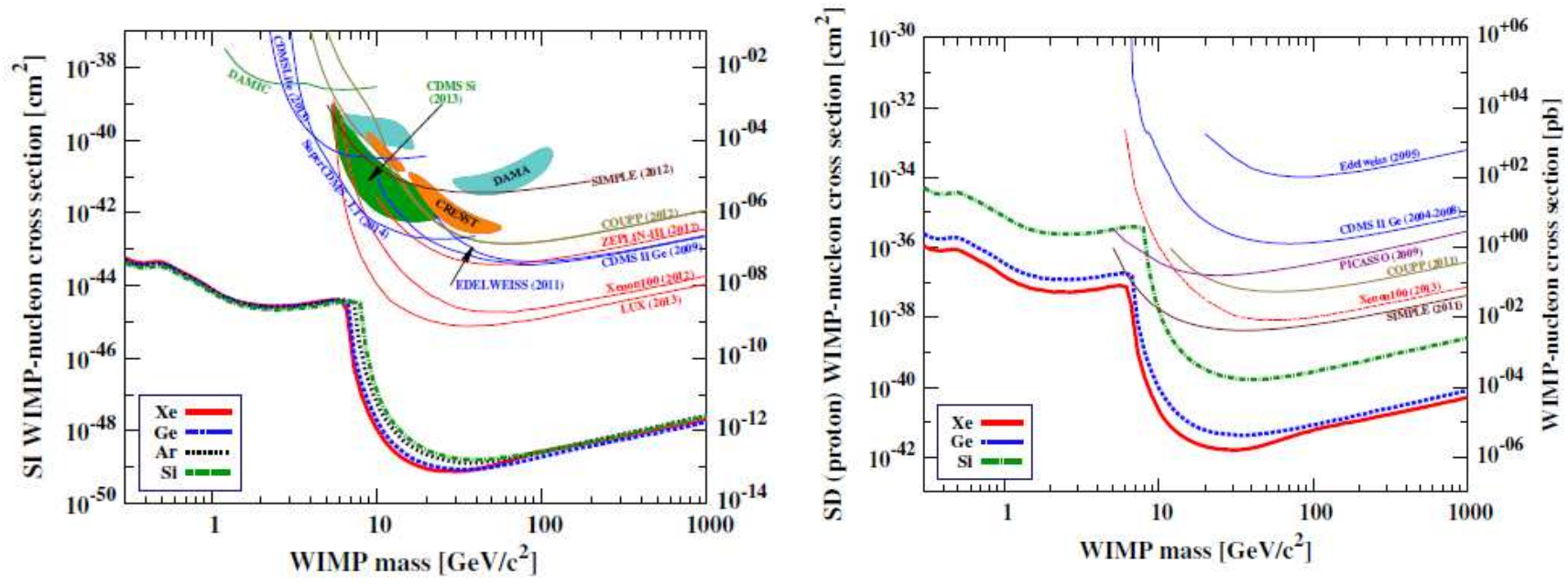

Fig. 2. The comparison of results obtained in different experimental setups, points to a significant difference of the spin-dependent SD and the spin-independent SI cross sections interactions $\sigma_{\mathrm{SD}} / \sigma_{\mathrm{SI}} \sim 10^{8}$ for simple working substances. (Diagrams are from [16]) and the spin-independent SI cross sections of interactions so that $\sigma_{\mathrm{SD}} / \sigma_{\mathrm{SI}} \sim 10^{8}$ for simple working substances.

Such results can be perceived if a non-contact interaction is mediated by magnetic type force. So let us fix on non-contact interaction of DM particles with working medium.

Above it was pointed, that the hypothetical candidate is a lightest supersymmetric particle, neutralino, which is a linear combination of Fermi super-partners of the photon, of the W-neutral boson and of the Higgs bosons, denoted as $\aleph=N_{11} \hat{B}+N_{12} \hat{W}_{3}+N_{13} \hat{H}_{1}^{0}+N_{14} \hat{H}_{2}^{0}$, where $N_{11}, N_{12}$, $N_{13}, N_{14}$ - are some constants (being the lightest supersymmetric particle, neutralino should be stable). Of course, being "neutral in all respects", neutralino has no electric charge, also if electroneutral elementary particles can possess a magnetic moment. Usually it occurs because of the reversible virtual transformation of the original "non-magnetic" particle (in ground state) to the multiplet partners, which have an electric charge (SU(2) baryons with isospin $1 \frac{1}{2}$ : (np)) or because of the existence of the virtual cloud of charged quanta of interaction field, which involves "naked nonmagnetic" particles. According to these modern concepts the neutron-magnetic moment is also formed (approximately).

Similarly, a very weak magnetic moment of neutrino $\left(\mu_{\mathrm{v}} \approx 10^{-13} \mu_{\mathrm{B}}\right)$ should occur [17] due to the electroweak processes illustrated by Feynman diagrams presented in Figure 3. 


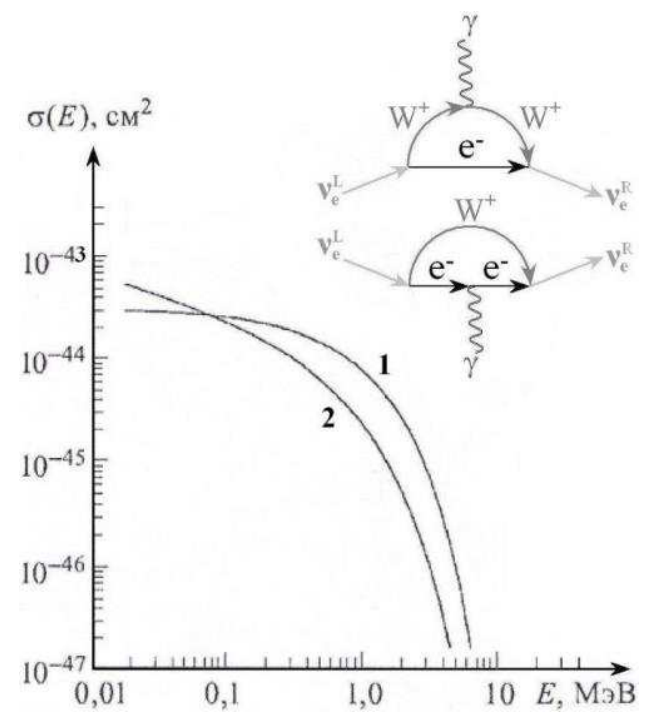

Fig. 3. Cross section of neutrino scattering on electron: 1 - the weak interaction (the

Weinberg's angle agrees with $\left.\sin ^{2} \theta_{W}=0,23\right) ; 2$ - magnetic interaction $\left(\mu_{v}=10^{-10} \mu_{B}\right)$ [17].

On insert: the Feynman diagrams, illustrating initiation of anomalous magnetic moment at Dirac (massive) neutrino $v_{(D)}$ are given.

In the framework of Salam-Weinberg theory of electroweak interactions (Standard Model) an electron neutrino $v_{\mathrm{e}}$ decays into an electron and $\mathrm{a}^{+}$boson with some non-zero probability and then through virtual particles annihilates for the time of $\Delta \mathrm{t} \approx \mathrm{h} /\left(\mathrm{m}_{\mathrm{W}} \mathrm{c}^{2}\right)$ turning into neutrino with another helicity. During the short $\left(\approx 2 \times 10^{-27} \mathrm{sec}\right)$ existence of the electric charged particles $\mathrm{e}^{-}$and $\mathrm{W}^{+}$, they have time to interact with an external electromagnetic field symbolized in the diagram by a photon $\gamma$. Thus the part of the radioactive corrections, which determines the energy shift, is interpreted as the interaction energy of the neutrino magnetic moment with the magnetic field.

A similar assumption about the presence of a magnetic type moment of DM particles is beyond the Standard Model, as in fact, DM does not exist inside the Standard Model as such. One of the channels is the reversible annihilation of the neutralino into a pair of a charged gauge boson W-type. A diagram illustrated the process shows that the two branches of virtual oppositely charged W-boson form a ring current $\mathrm{I}_{\mathrm{W}}$, whose corresponding boson loop has the same

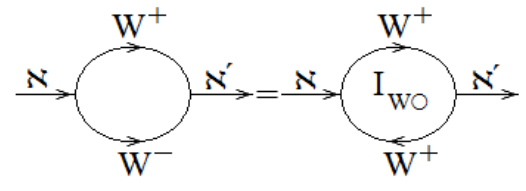
charge (and effective area of $\mathrm{S}_{\mathrm{W}}$ ).

We express the magnetic moment of the boson loop $S_{W \circ} I_{W \circ}$ through the square of the Compton wavelength $S_{W o} \approx \hbar^{2} /\left(m_{W} c\right)^{2}$ and, using the Heisenberg relations, we estimate the loop current $I_{W \circ} \approx e / \tau_{W} \approx e m_{W} c^{2} / \hbar$. Get in the end the expression $S_{W \circ} I_{W \circ} \approx e \hbar / m_{W}=\mu_{W} \quad$ coincides with the structure of the standard formula of the Bohr magneton $\mu_{B}$ and differs from the last replacing 
the electron mass $m_{e}$ on $m_{W} \approx 1,6 \times 10^{5} m_{e}$. Accordingly $\mu_{W}$ is approximately 5 orders of magnitude smaller $\mu_{B}$. The magnetic field with induction $B \approx 10 \mathrm{~T}$ offers energy the action on $\mu_{\aleph}$ at the level of $\mu_{W} B \approx 4 \times 10^{-9} \mathrm{eV}$. The probability of interaction between the atom adsorber, whose atomic orbital current induces a field $B_{o r b} \approx 10 \mathrm{~T}$ (typical value field for the spin-orbit effects) with the magnetic moment of the boson loop occurring during reversible decay of the neutralino, estimate, squaring the corresponding amendment $\delta \psi_{W \circ}$ to the amplitude of the unperturbed boson loop $\psi_{W \circ}=\psi_{W \circ}^{(0)}+\delta \psi_{W \circ}=\psi_{|W \circ\rangle}^{(0)}+\sum_{\forall|W \circ\rangle^{\prime}} \frac{\mu_{W} B_{o r b}}{E_{|0\rangle}-E_{|W \circ\rangle^{\prime}}} \psi_{|W \circ\rangle^{\prime}}^{(0)}$. Thus, the probability sought for a typical value of the energy, eventually lost by neutralino $\delta E_{\aleph} \approx 40 \mathrm{eV}$ (the absorber is transferred in a reliable registration), is estimated as $\left(\mu_{W} B_{o r b} / \delta E_{\aleph}\right)^{2} \approx 10^{-20}$. Lined up in a linear chain of $10^{20}$ absorber atoms and adding the probabilities of magnetic interaction with all atoms, bringing its level to the level of confidence $\sum_{1}^{10^{20}} 10^{-20} \cong 1$. The length of this "chain of complete absorption" is $a \times 10^{20}$ where $a$, a solid-state atomic absorption period. "Build" a hypothetical absorber of a large number of such chains, and let his face is square $S_{\mathbf{r}}$. The cross section of the magnetic interaction

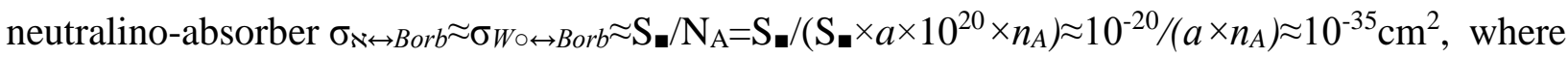
$n_{A} \approx 3 \times 10^{22} \mathrm{~cm}^{-3}$ - the concentration of atoms in the absorber. Besides fact implies that the less registered by the energy, the more such events should occur, and the higher is the estimated section.

Estimate «magnetic cross section» at the level of $10^{-35} \mathrm{~cm}^{2}$, happens to be noticeably higher typical values of level of $10^{-44} \mathrm{~cm}^{2}$, that in the case of its justice indicates character of optimal experimental design experiment on search of dark matter particles as the neutralino: it is required calorimeter as possible low energy detection threshold (not worse $\delta \mathrm{E} \approx 40 \mathrm{eV}$ ), a solid-state absorber which is to be made of atoms with strong spin/orbital effect, indicating the presence of a large (not lower $\mathrm{B}_{\text {orb }} \approx 10 \mathrm{~T}$ ) orbital magnetism. On a calorimeter role with energy threshold of order $\delta E \approx 40 \mathrm{eV}$ would be able pretend nigh only the unopposed candidate - the system of SQUID- $\{$ the paramagnetic absorber $\}$ [18, 19]. This cryogenic system (Fig. 4) consists of paramagnetic absorber demagnetizing due to heating the detected radiation $\delta E$ and quantum interferometer, fixing a corresponding decrease in the magnetic moment of the absorber $\delta m_{a b s}$.

At sufficiently low $(T \approx 1 \mathrm{~K})$ temperatures when the heat capacity of paramagnetic material prevails the magnetic contribution $\delta E \approx B \delta m_{a d s}$ (where $B$, depending on the mode of operation of the system or the induction of external magnetizing field [18], or the residual field paramagnet [10]). The variation of magnetic flux, directly registered by the SQUID is $\delta \Phi \approx \mu_{0} \delta m_{a d s} / h \approx \mu_{0} \delta E$ $/(h B)$, where $h$ - the absorber length (the height of paramagnetic cylinder), $\mu_{0}=4 \pi \times 10^{-7} \mathrm{H} / \mathrm{m}$. 
Superconducting quantum interferometer (SQUID) [20 - 22] due to the sensitivity of their Josephson tunnel junctions to the difference between the Cooper condensate quantum phase [23], the incoming in operational superconducting SQUID ring under the influence of the magnetic field detected, fixes flow variations in the ring as a fraction of the basic period - flux quantum $\Phi_{0}=$ $\pi \mathrm{h} / \mathrm{e} \approx 2,07 \times 10^{-15} \mathrm{~Wb}$ (which corresponds to a phase change $\delta \varphi=2 \pi$ ). At the same time a good but not record sensitivity of the modern interferometer is considered the value $\delta \Phi \approx 10^{-6} \Phi_{0} / \sqrt{ } \mathrm{Hz}$.

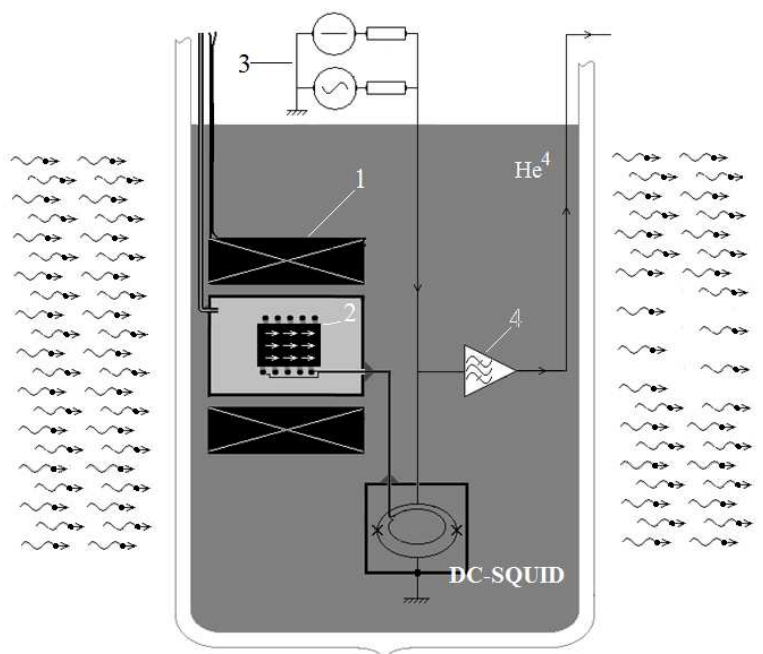

Fig. 4. Schematic view of the system of SQUID- $\{$ the paramagnetic absorber $\}: \mathbf{1}$ - the superconducting solenoid magnetization; $\mathbf{2}$ - the paramagnetic absorber magnetic; $\mathbf{3}$ - current generators, 4 - narrow-band low-frequency amplifier.

This value corresponds the energy resolution $\delta E \approx h B \delta \Phi / \mu_{0} \approx 2 \times 10^{-18} \mathrm{~J} / \sqrt{ } \mathrm{Hz} \approx 15 \mathrm{eV} / \sqrt{\mathrm{Hz}}$ ( $h \approx 0,1 \mathrm{~m}, B \approx 0,01 \mathrm{~T}$ ), that makes it possible to fix $\delta E \approx 40 \mathrm{eV}$ with maximal frequency nearly 3 events per second. However, with the expected Earth conditions in the flux density of a DM particle at the level of no more than $200 \mathrm{~km} / \mathrm{s} \times 1500$ piece $/ \mathrm{m}^{-3}=3 \times 10^{8} \mathrm{~s}^{-1} \mathrm{~m}^{-2}$ to the absorber with strong paramagnetic atomic orbital magnetism containing at sizes $h \times S \approx 0,1 \mathrm{~m} \times 0,01 \mathrm{~m}^{2}$ approximately 0,15 kmole $\approx 10^{26}$ atoms with a cross section of interaction $\sigma \approx 10^{-35} \mathrm{~cm}^{2}$ ensure maximum registration rate of $3 \times 10^{-5}$ events/s $\approx 4$ events/day. Thus, the margin of recording rate of about 6 orders of magnitude $\left(10 / 3 \times 10^{-5}\right)$ can be used to compensate for the loss of sensitivity of the system associated with a low $(<1$, depending on the design [22]), the transmission coefficient $K$ of the superconducting flux transformer, which provides communication macroscopic working body of absorber with a microscopic phase-sensitive ring SQUIDa where the Josephson junctions (such compensation possible to the level of $\left.K \approx\left(3 \times 10^{-5} / 10\right)^{1 / 2} \approx 0,0017\right)$. 
Features of the various modes of operation of SQUID -\{paramagnetic absorber $\}$ system discussed in detail in the works: [24] (direct measurement of the growth of entropy using the method of adiabatic demagnetization), [25] (sensitization by replacing atom paramagnetism a nuclear with cooling the dissolution refrigerator $\mathrm{He}^{3}$ in $\mathrm{He}^{4}$ ), [26] (dual-channel mode to eliminate lepton processes), [27] (estimate sensitivity in strong fields saturation asymptotic methods of statistical mechanics), [28] (resonance registration $\mathrm{THz}$ radiation with a wavelength near 10mkm).

Specificity of the magnetic type interaction of DM particles with "normal" matter is its "tangential character" (as opposed to prior conventional interaction "nuclear/head-on" type). Currently very popular are also considering the theory of Dark Energy (hypothetical pervasive substance responsible for additional relative acceleration of the Hubble law recession of galaxies) and elementary dark matter particles of common general physical positions. As an example of such a concept can be seen in fact dark energy as unperturbed state of the all-pervading "Dark Substance" density of about $300 \mathrm{TeV} / \mathrm{m}^{3}$, which swings (alike acoustic quanta in the gas or liquid) play the role of elementary particles of Dark Matter.

\section{Conclusion}

In this paper we have analyzed the problems of DM particles registration. The DM conundrum stands in the same row with other contemporizing physical challenges: existence of Dark Energy, revealing axions [29], detection of gravitational waves [30], and comprehension the mass of neutrino. The possible solution is laid in acceptance the feature of a new kind of matter and/or new type of interaction. As was stated the usage of SQUIDs can help to detect low probability acts of interaction not only in head-on interactions [31], but in "tangential character" interaction also.

\section{References}

1. Zwicky F. (1933). Helv. Phys. Acta, 6, 110.

2. EROS Collaboration (2002). Astron. Astrophys., 389, L69.

3. Straumann N. (2006). arXiv, hep-ph/0604231.

4. Penzias A.A., Wilson R.W. (1965). ApJ , 142(1), 419-421.

5. Eisenstein J.D., Hu W. (1997). Power Spectra for Cold Dark Matter and its Variants. Astrophys.J., 511.

6. Jungmann G., Kamiokowsky M., Griest K. (1996). Phys Rep., 267, 195.

7. Berezinsky V., Dokuchaev V., Eroshenko Yu. (2006). Phys.Rev., D, 73, 063504. 
Proceedings of International Conference PIRT-2015

8. Ryabov V.A., Tsarev V.A., Tskhovrebov A.M. (2008). Phys. Usp., 51, 1091-1121.

9. Ellis J., Hagelin J., Nanopoulos D. (1984). Nucl. Phys., B, 238, 453.

10. Servant G., Tait T. (2003). Nucl. Phys., 650, 391

11. Shi J., Wang H., Mo H.J. (2015). ApJ, 807, 37.

12. Broadhurst T., Molnar S. (2015). ApJ, 800, 37.

13. Harvey D., Massey R., Kitching T., Taylor A., Tittley E. Science, Vol. 347, no. 6229, 14621465.

14. Cantalupo S., Arrigoni-Battaia F., Prochaska J.X., Hennawi J.F., Madau P. (2014). Nature, $506,63-66$.

15. Massey R. (2015). The behaviour of dark matter associated with four bright cluster galaxies in the $10 \mathrm{kpc}$ core of Abell 3827. Monthly Notices of the RAS.

16. Ruppin F., Billard J., Figueroa-Feliciano E., Strigari L. (2014). PR, D, 90, 083510.

17. Durbins A.V. (2001). Elementary-particle physics and atomic nucleus, 32 (3), 734-749.

18. Buhler M., Umlauf E. (1993). J. Low Temp. Phys., 93, 697-702.

19. Zherikhina L.N., Golovashkin A.I., Mishachev V.M., Troitskij V.F., Tskhovrebov A.M. (2003). Journal the Applied Physics, №6, 27-34.

20. Barone A., Paternò G. (1982). Physics and applications of the Josephson effect. New York: Wiley.

21. Golovashkin A.I., Elenskij V.G., Likharev K.K. (1983). Josephson effect and its application. Moscow: Nauka [Science].

22. Clarke J., Braginski A.I. (2006). The SQUID Handbook (vol.1,2). New York:Wiley.

23. Josephson B.D. (1962). Phys. Lett., 1, 251.

24. Golovashkin A.I., Izmailov G.N., Zherikhina L.N., Kuleshova G.V., Tskhovrebov A.M. (2006). Quantum Electron, 36 (12), 1168-1175.

25. Golovashkin A.I., Izmaïlov G.N., Kuleshova G.V., Khánh T.Q., Tskhovrebov A.M., Zherikhina L.N. (2007). EPJ B, 58(3), 243-249.

26. Golovashkin A.I., Izmaïlov G.N., Ryabov V.A., Tshovrebov A.M., Zherikhina L.N. (2013). Amer. J. of Mod. Phys., 2(4), 208-216.

27. Izmaïlov G.N., Zherikhina L.N., Tshovrebov A.M. (2015). Measurement techniques, №8, 317.

28. Dresvyannikov M.A., Karuzskii A.L., Perestoronin A.V., Tskhovrebov A.M., Zherikhina L.N. (2014). Photoresponse Beyond the Red Border of the Internal Photoeffect (Designing Problems of Photon Counting Schemes in 10 Mu M Band). Proceedings of SPIE, 9440, 
Proceedings of International Conference PIRT-2015

29. Gorelik V.S., Izmaïlov G.N. (2011). Bull. Lebedev Phys Inst., 38, No. 6, 177-183.

30. Pustovoit V.I., Morozov A.N., Gladyshev V.O., Izmaïlov G.N. (2015). Laser gravitational waves antennae: Short review. Moscow : BMTSU.

31. Izmaïlov G.N. (2008). Measurement Techniques, 51, No. 11, 1171-1177. 